\title{
London Deauville Criteria Point Scale 3
}

National Cancer Institute

\section{Source}

National Cancer Institute. London Deauville Criteria Point Scale 3. NCI Thesaurus. Code C99748.

The results of the positron emission tomography are negative; the tracer uptake is greater than the mediastinum but less than or equal to the liver. 